\title{
Evaluación e intervención de los problemas de Ansiedad y Depresión en Atención Primaria: Un problema sin resolver
}

\author{
a Universidad de Castilla-La \\ Mancha, Albacete, España. \\ bUniversidad de Málaga, \\ España. \\ c Universidad Complutense de \\ Madrid, España. \\ Correspondencia: José Miguel \\ Latorre Postigo, Departamento \\ de Psicología, Facultad de \\ Medicina, c/ Almansa $\mathrm{n}^{\circ} 14$ \\ 02008 - Albacete. Correo \\ electrónico: Jose.Latorre@ \\ uclm.es.
}

Recibido el 13 de enero de 2012.

Aceptado para su publicación el 23 de enero de 2012.
José Miguel Latorre Postigo ${ }^{\mathrm{a}}$, Beatriz Navarro Bravo ${ }^{\mathrm{a}}$, Marta Parra Delgado ${ }^{\mathrm{a}}$, José M. Salguero ${ }^{b}$, Cristina Mae Wood ${ }^{c}$, Antonio Cano Vindel ${ }^{\mathrm{c}}$

\begin{abstract}
RESUMEN
Los problemas de salud mental, especialmente los de ansiedad y depresión, suponen aproximadamente una de cada cuatro consultas que reciben los médicos de atención primaria. En este trabajo se presenta una revisión que pretende responder a dos preguntas interrelacionadas. En primer lugar, ¿cómo abordan los médicos de atención primaria el diagnóstico y el tratamiento de la ansiedad y depresión? Y en segundo lugar, ¿cómo se puede solucionar el problema? Para responder a la segunda pregunta vamos a tratar los siguientes aspectos: las necesidades de formación de los médicos de atención primaria, el desarrollo de instrumentos de evaluación específicos para su uso en ese contexto, el desarrollo de técnicas psicoterapéuticas adaptadas a las características de la atención primaria, así como el incremento del número de profesionales sanitarios dedicados a la salud mental.
\end{abstract}

Palabras clave. Atención Primaria, Ansiedad, Depresión.

\section{ABSTRACT}

Assessment and intervention of Anxiety and Depression disorders in Primary Care: An unsolved problem

The mental health problems, especially anxiety and depression, represent approximately one in every four visits received by primary care physicians. In this paper we present a review that aims to answer two interrelated questions. First, how primary care physicians diagnose and treat anxiety and depression? And secondly, how can we solve the problem? To answer the second question we will discuss the following aspects: the training needs of primary care physicians, the development of specific assessment tools for being used in this context, the development of psychotherapeutic techniques adapted to the characteristics of the primary care setting as well as the increasing number of health professionals dedicated to mental health.

Key words. Primary Care, Anxiety, Depression.

\section{EL PROBLEMA}

En todo el mundo, los problemas de salud mental suelen ser atendidos por primera vez más en las consultas de atención primaria (AP) que en las de salud mental ${ }^{1,2}$. En España, al menos en una de cada cinco consultas que recibe un médico de familia, el paciente va por algún problema de índole psicológica o psiquiátrica, ya que diversas investigaciones señalan que la prevalencia de psicopatología en AP se mueve entre un $22 \%$ y un $29 \%$, pudiendo llegar incluso al $45 \%$ en algún estudio ${ }^{3-9}$. Los datos parecen apuntar que existe una asociación entre la enfermedad mental y el alto número de visitas al médico de medicina familiar ${ }^{10}$, de manera que los pacientes con trastornos mentales o conflictos psicosociales crónicos tienen tendencia a consultar de forma repetida en los diversos dispositivos de $\mathrm{AP}^{11}$.

Como ya hemos señalado en otro momento ${ }^{12-14}$, ningún grupo de enfermedades ha experimentado un cambio más importante en su detección y tratamiento como los trastornos de salud mental en AP. Ya en los años sesenta, los trabajos originales de Sheppard, Cooper, Brown y Kalton en el Reino Unido ${ }^{15}$, cuyos hallazgos básicamente continúan siendo válidos en la actualidad, indicaron que más de un $15 \%$ de los pacientes que consultan en AP presenta un trastorno psiquiátrico, y que de ellos sólo el $5 \%$ se deriva a salud mental. 
En los años setenta y ochenta los estudios confirmaron estos primeros hallazgos, a la vez que se fueron sofisticando desde el punto de vista metodológico. En este sentido, se realizaron diseños en dos fases empleando instrumentos como el General Health Questionnaire (GHQ) en la primera fase y entrevistas psiquiátricas estructuradas, como el Present State Examination (PSE) o la Composite International Diagnostic Interview (CIDI), en la segunda. Este tipo de estudios permitió conocer la prevalencia y características clínicas de la mayoría de los trastornos psiquiátricos en AP. En nuestro país también se realizaron estudios epidemiológicos similares que permitieron obtener datos definitivos en este sentido y que apenas diferían de los estudios internacionales. Concretamente, las cifras de trastornos mentales en España varían entre el $19 \%$ y el $46 \%$ de unos estudios a otros (para una revisión ver Baca et al., 2001) ${ }^{16}$.

Con los datos más recientes en la mano, se puede decir que los trastornos psiquiátricos están presentes en un alto porcentaje de los pacientes que acuden a las consultas de AP o de otros especialistas no psiquiatras. Además, los trastornos psiquiátricos se presentan con frecuencia en pacientes con diferentes enfermedades neurológicas, cardiovasculares o pulmonares $^{8}$. En este sentido Wells, Golding y Burnam ${ }^{17}$ mostraron que la prevalencia en 6 meses de trastornos psiquiátricos fue el doble en pacientes con enfermedades neurológicas o cardiacas (35$38 \%$ ) que en pacientes sin procesos orgánicos. Además, cuando la depresión o la ansiedad van asociadas a una patología crónica, el pronóstico en la recuperación a un año suele ser menor que cuando se presenta sola ${ }^{18}$.

La prevalencia de la ansiedad y depresión en AP varía de unos estudios a otros, y de unos países a otros, situándose entre el 15 y el $30 \%$. Por ejemplo, en un estudio realizado por investigadores de la Universidad de Budapest (Hungría), se muestra una prevalencia de $12,5 \%$ en el último mes y un $16,8 \%$ en el último año ${ }^{19}$.

En el estudio más reciente, sobre la prevalencia de la ansiedad en AP, se entrevistó a 965 pacientes, de los cuales el 19,5\% (IC 95\% 17,0-22,1) tenía al menos un trastorno de ansiedad, el 8,6\% (IC $95 \%$ 6,9-10,6) trastorno de estrés postraumático, el $7,6 \%$ (IC 95\% 5,9-9,4) trastorno de ansiedad generalizada, el 6,8\% (IC 95\% 5,3-8,6) trastorno de pánico, y el 6,2\% (IC 95\% 4,7-7,9) fobia social. También se observó que a mayor número de diagnósticos mayor fue el deterioro de la calidad de vida $(p=0.0001)$. De todos los pacientes que podían ser diagnosticados de algún tipo de trastorno por ansiedad, el $41 \%$ no estaba siguiendo ningún tratamiento ${ }^{20}$.

\section{Infradiagnóstico}

Probablemente la demanda real por problemas de salud mental sea superior a la demanda percibida por los médicos debido a las dificultades que éstos tienen para detectar los trastornos psiquiátricos. En nuestros datos se observa cómo los médicos de atención primaria (MAP) manifiestan, con bastante frecuencia, dificultades en la identificación y evaluación de este tipo de trastornos. Dichas dificultades son más frecuentes cuando se presentan pacientes con trastornos somatomorfos, trastornos de la alimentación y demencias ${ }^{21}$.

Por tanto, uno de los problemas que se presenta en AP es el infradiagnóstico de los trastornos mentales por parte de los médicos, pues casi la mitad de estos pacientes no han sido diagnosticados y no reciben tratamiento para dichos trastornos ${ }^{22}$. En el estudio longitudinal sobre los trastornos de ansiedad en AP denominado Primary Care Anxiety Project, llevado a cabo en EEUU, un gran número de pacientes de 15 centros de AP completaron un cuestionario de cribado de síntomas de ansiedad y los que dieron positivo fueron invitados a realizar una entrevista diagnóstica, completando dicha entrevista 1.634 pacientes para diagnosticar un trastorno mixto de ansiedad-depresión, entre otros desórdenes mentales. La muestra de pacientes que tenían al menos un trastorno de ansiedad quedó formada por 539 participantes ${ }^{23}$, de los cuáles el $47,3 \%$ no fueron tratados, el $21 \%$ recibía exclusivamente medicación para problemas mentales, el 7,2\% recibía solamente terapia y el $24,0 \%$ recibía ambos tipos de tratamiento. El tratamiento que se les proporcionaba, aunque con algunas diferencias, era similar al que se podía proporcionar en los servicios especializados de salud mental.

En un trabajo realizado en un centro de AP de Barcelona centrado en el estudio de la depresión se concluía que existe una elevada prevalencia de este trastorno. Según este estudio permanece infradiagnosticado un $44,3 \%$ de las personas con probable trastorno depresivo (sobre todo mujeres, viudos, jubilados, los que han presentado acontecimientos vitales estresantes y los individuos más frecuentadores) ${ }^{24}$.

En general, se puede decir que los MAP sólo detectan la mitad de los casos de ansiedad y/o depresión. En sendos artículos publicados en prestigiosas revistas médicas como Lancet ${ }^{22,25,26}$, se alerta de las dificultades de los MAP a la hora de detectar los casos de depresión. Concretamente a raíz de la aparición en Lancet del artículo de Mitchel et al..$^{26}$ se publicó en el mismo número un editorial con el título: 
¿Son los médicos generalistas realmente incapaces de diagnosticar la depresión? El citado trabajo supone un referente en esta área de investigación, ya que se trata de un meta-análisis en el que se han analizado 118 estudios, incluyéndose finalmente 41 de ellos con un total de más de 50.000 pacientes. Los resultados apuntan a que los MAP detectan el $47,3 \%$ de los casos de depresión y solo registran el $33,6 \%$ de los casos. La sensibilidad es de 50,1 y la especificidad de 81,3 . La relación entre verdaderos positivos y falsos negativos se mantiene constante, independientemente de la prevalencia real de la depresión en cada uno de los estudios. Así, cuando la prevalencia en la población estudiada es del 10\% se detecta el $5 \%$. Y cuando es más alta $(20 \%)$ se diagnostican correctamente la mitad (10\%).

\section{Sobrecarga asistencial}

Una cuestión que afecta claramente a las posibilidades de diagnóstico e intervención sobre los problemas de ansiedad y depresión en AP es la sobrecarga asistencial y el poco tiempo del que se dispone para dedicarle a estos casos. En un trabajo reciente se muestra que la mayoría de los MAP $(75,3 \%)$ atiende a 35 o más pacientes cada día. Asimismo, la mitad de los médicos $(50,8 \%)$ tiene asignados más de 1.500 pacientes. Todo esto conlleva un tiempo medio por consulta muy inferior a los diez minutos ${ }^{21}$. Y si tenemos en cuenta la problemática asociada a los trastornos de ansiedad y/o depresión, ese tiempo aún debería ser mayor. En esta cuestión los médicos están claramente de acuerdo: no pueden hacerse cargo de la demanda de problemas de salud mental con la sobrecarga de trabajo actual ${ }^{21}$. En un estudio sobre la duración de las consultas de $\mathrm{AP}^{27}$ el tiempo dedicado a cada consulta varió según el tipo de problema atendido, siendo de 8,6 minutos (IC 95\% 6,1-11) para los problemas psicosociofamiliares, de 7,1 minutos (IC $95 \% 6,6-7,7)$ para los orgánicos y de 6,9 minutos (IC 95\% 5,2-8,4) para los burocráticos $(p<0,05)$. En otro trabajo, realizado en seis países europeos (Alemania, España, Reino Unido, Holanda, Bélgica y Suiza) sobre la duración de las consultas en AP, se puede constatar que España es, después de Alemania, el país donde menos duran las consultas. Mientras la media de los seis países es de 10,7 minutos, en Alemania es de 7,6 y en España de $7,8^{28}$.

Con los datos disponibles se puede decir que los problemas de salud mental (fundamentalmente ansiedad, depresión y somatización) son los que mayor carga de trabajo les produce a los MAP. En este sentido, un estudio clásico de Vázquez-Baquero et al. ${ }^{29,30}$ encontró que la morbilidad psiquiátrica es el principal determinante de la utilización de las consultas de AP en ambos sexos y que una de cada seis consultas en los hombres y una de cada cinco en las mujeres están relacionadas con un problema de salud mental. Además, los resultados muestran una asociación entre trastornos mentales y mayor número de visitas al $\mathrm{MAP}^{10}$ tanto por los propios problemas de salud mental como por otras causas. En resumen, se podría decir que el paciente que tiene problemas de ansiedad y/o depresión es un "hiperfrecuentador", ya que los datos señalan que la prevalencia de ansiedad y/o depresión en los pacientes que más frecuentan las consultas de AP es entre el $40 \%$ y el $60 \%$. Los factores que mejor predicen la hiperfrecuentación en pacientes con problemas de ansiedad y depresión son: frecuentación previa, historial psiquiátrico en el pasado, presencia de dificultades sociales, nivel presente de consumo de alcohol, puntuación total de síntomas psiquiátricos y la puntuación total en ansiedad $^{31}$.

En definitiva, el poco tiempo disponible para atender a los pacientes en AP, unido a que los problemas de salud mental requieren más tiempo, supone un factor determinante a la hora de explicar las dificultades que pueden tener los médicos de AP a la hora de atender esa demanda ${ }^{21}$.

\section{Razones para explicar las dificultades con el diagnóstico}

Además de la sobrecarga asistencial y la falta de tiempo, se han aducido otras razones para explicar estas dificultades en el diagnóstico de los trastornos mentales en $\mathrm{AP}^{16,32-34}$.

En primer lugar, la poca familiaridad con los trastornos psíquicos, pues existe un amplio consenso en que es necesario mejorar las habilidades de los MAP en el diagnóstico y tratamiento de los trastornos mentales ${ }^{35}$ y nuestros datos confirman precisamente la necesidad de ampliar su formación en salud mental ${ }^{21}$.

En segundo lugar, la mayoría de los pacientes con trastornos mentales que acuden al MAP consultan por síntomas somáticos (insomnio, cefalea, fatiga, pérdida de peso, etc.) que pueden ser atribuidos a enfermedades físicas en lugar de poner al médico sobre la pista de un posible trastorno mental (Baca et al., 2001) y, de hecho, cuando el paciente realiza la consulta por un motivo psicológico, la detección de trastornos mentales mejora considerablemente ${ }^{34}$. Además conviene recordar que un $6,7 \%$ de los pacientes de AP presentan diagnóstico de hipocondría en el momento presente ${ }^{36-38}$ y, en general, nos encontramos con una alta tasa de prevalencia de los trastornos por somatización. En un estudio reciente el 50,3\% (Criterios ICD-10) de los pacientes de AP han tenido alguna vez en la vida un trastorno somatoforme, y el $25,6 \%$ lo 
padecen en el momento actual ${ }^{39}$. Además, más de la mitad de los que padecen trastornos somatomorfos en el momento actual, presentan al mismo tiempo depresión (5,3\%), ansiedad (2,3\%) o ambas $(6,1 \%)$. Desde esta perspectiva, el énfasis se sitúa en el proceso de somatización, es decir, de presentación de la enfermedad psiquiátrica (depresión y/o ansiedad) en forma de síntomas somáticos (lo que se denomina "presentación somatizada") y no en forma de síntomas psicológicos ("presentación psicológica"), algo que ocurre con frecuencia en el contexto de $\mathrm{AP}^{40,41}$.

Por último, las dificultades que encuentra el médico de familia para distinguir entre ansiedad y depresión cuando ambos trastornos aparecen de manera simultánea en el enfermo ${ }^{33,42,43}$. En relación con esta dificultad, los médicos encuestados opinan que tienen un conocimiento alto tanto de los trastornos de ansiedad como de los del estado de ánimo ${ }^{21}$. Esta autoevaluación positiva sobre el nivel de conocimientos acerca de estos trastornos debería de correlacionarse con un nivel aceptable en el diagnóstico de los mismos en AP. No obstante, diferentes estudios muestran que, por ejemplo, los trastornos depresivos permanecen infradiagnosticados entre un tercio y la mitad de los casos en el contexto de la AP $32,44,45$.

En este sentido, en la mayoría de los pacientes de AP con depresión mayor (DM) coexisten otros trastornos mentales, frecuentemente trastornos de ansiedad. En un estudio reciente ${ }^{46}$ se observa que, al mismo tiempo que DM, coexisten en un $55,2 \%$ (IC 95\% 41,6-68,8) el trastorno de ansiedad generalizada, en un $33,8 \%$ (IC 95\% 21,1-47,1) el trastorno de pánico, en un 15,7\% (IC 95\% 10,323,4 ) la distimia y en un $6,6 \%$ (IC 95\% 3,3-12,8) los trastornos por somatización. Además en este trabajo se comprobó que solo un tercio de los pacientes con DM recibía tratamiento específico con antidepresivos. Sin embargo, la proporción de consumo de ansiolíticos es mayor (1/2), tanto en los que presentan comorbilidad como en los que no.

\section{Tratamiento de ansiedad y depresión en AP y la adherencia terapéutica}

En la literatura sobre el tema se ha puesto de manifiesto reiteradamente que una gran proporción de las personas con problemas de salud mental permanece sin tratamiento $o$ con un tratamiento inadecuado. Un trabajo reciente, llevado a cabo con los datos de España $(n=5.600)$ del European Study of the Epidemiology of Mental Disorders (ESEMeD), analizó una submuestra de 133 sujetos que habían seguido algún tipo de tratamiento para la ansiedad o depresión en el año anterior en AP o en los servicios especializados en salud mental. La proporción de pacientes que recibió un tratamiento mínimamente adecuado fue similar en los servicios especializados y en AP $(31,8 \%$ y 30,5\%, respectivamente). En resumen, sólo la tercera parte de los casos de ansiedad o depresión que son tratados en España cumplen los requisitos mínimos basados en la evidencia ${ }^{47}$.

Como ya hemos apuntado, la mayoría de los estudios señalan que tanto los MAP como los de otras especialidades detectan sólo la mitad de los pacientes que sufren estos trastornos ${ }^{22}$. Sin embargo, cuando se produce algún tipo de intervención, el tratamiento que reciben de los MAP o de los especialistas produce resultados similares ${ }^{23}$.

Uno de los problemas que con más frecuencia se han citado, en relación con el tratamiento de los trastornos de ansiedad y/o depresión, es la falta de adherencia terapéutica ${ }^{48}$. Desde los estudios clásicos de Ley ${ }^{49}$ se sabe que entre los factores que mejor predicen el cumplimiento de las recomendaciones médicas se encuentra la buena comunicación con el médico. La escasez de habilidades de comunicación con los pacientes se agrava con la falta de tiempo dedicado a las consultas. Y en el caso de los trastornos mentales esta cuestión supone un problema mayor. En este sentido, en un estudio reciente sobre los factores determinantes de la duración de las consultas de $\mathrm{AP}$ en seis países europeos ${ }^{28}$ se puso de manifiesto que, cuando paciente y/o médico perciben que la consulta está relacionada con un problema de salud mental, el tiempo medio se incrementa considerablemente.

\section{LAS SOLUCIONES}

Este es el apartado más difícil de completar. Por un lado, se puede decir que ya existen soluciones basadas en la evidencia, como la mejora del diagnóstico o la intervención en base a las tecnologías de la información y comunicación (TIC). Pero, por otro, existen otras soluciones que, aunque no se ha probado su eficacia todavía de manera sistemática, puede suponerse que serán efectivas. En cualquier caso podemos decir que las soluciones se pueden agrupar en: a) incremento de la formación de los MAP en relación con los problemas de ansiedad, depresión y otros trastornos relacionados como la somatización - la dependencia a las benzodiacepinas; b) desarrollo de nuevos instrumentos de evaluación y diagnóstico de la depresión y/o ansiedad, útiles en el contexto de AP, así como el perfeccionamiento de los ya disponibles; c) desarrollo y adaptación de técnicas psicoterapéuticas al contexto de la AP; d) por supuesto, una reorganización de los sistemas de $A P$ que incluya un aumento del tiempo medio por consulta (mínimo 10 minutos) e incorpore a 
otros profesionales como psicólogos o enfermeras especialistas en salud mental a los equipos de AP.

\section{Incremento de la formación específica de los MAP}

En relación a la necesidad de incrementar la formación, parece ser que el aumento progresivo de la demanda percibida por parte de los médicos de atención primaria se debe a un mayor conocimiento de estos especialistas de la patología mental y a una desmitificación progresiva de dicha patología, lo que facilita su detección y abordaje. Los médicos encuestados en un trabajo reciente son conscientes de ello, de forma que, cuando se les preguntaba qué recursos piensan que se deberían incluir para satisfacer la demanda existente en problemas de salud mental en AP, la mayoría opina que debería existir una mayor formación específica en materia de salud mental. La razón de este incremento de formación específica creemos que tiene que ver con la opinión mayoritariamente expresada de que "una parte sustancial de los problemas psíquicos y de las acciones en el campo de la salud mental deben ser resueltas por los equipos de $\mathrm{AP}$, sin que requieran la presencia directa de personal especializado"21.

Como se ha señalado en diferentes trabajos, la capacidad de diagnosticary tratar de forma adecuada los problemas derivados de ansiedad y depresión está relacionada con el nivel de formación en la materia de los MAP21,22,34,50. Además, en algunos trabajos de evaluación de programas educativos para mejorar la capacidad de diagnóstico de los MAP se ha comprobado que, tras la intervención, la capacidad de detectar correctamente los trastornos de ansiedad y/o depresión se incrementa de manera significativa ${ }^{51-53}$. En definitiva, el nivel de formación desempeña un papel importante en el correcto diagnóstico y de éste depende el plan de tratamiento, que en la mayoría de los casos suele ser farmacológico, y los estudios que prueban la eficacia de los fármacos están hechos con pacientes que tienen un diagnóstico claro. Además, no conviene olvidar los problemas ya aludidos en torno a los efectos secundarios de los psicofármacos. En este sentido, en un trabajo de Latorre et al..$^{50}$ sobre el manejo de las adicciones por parte de los MAP, solamente un $19,5 \%$ de los médicos encuestados afirma tener un alto grado de conocimiento sobre la adicción a las benzodiacepinas, a pesar de estar tan extendida la prescripción de este tipo de fármacos en el contexto de $\mathrm{AP}^{54}$.

En consecuencia, se hace cada vez más evidente la importancia de mejorar la capacidad de los médicos no psiquiatras para detectar las enfermedades mentales, ya que una vez detectadas el tratamiento suele ser efectivo. Para conseguirlo se hace necesaria la adopción de nuevas estrategias como 41 el desarrollo de programas educativos dirigidos a los médicos de AP y la implantación de instrumentos de diagnóstico de enfermedades mentales que puedan ser utilizados por los no especialistas. La importancia de un correcto diagnóstico es crucial, ya que en la mayoría de los casos los médicos de AP suelen optar por la prescripción de fármacos para el tratamiento de los problemas de ansiedad y/o depresión. Y los estudios que prueban la eficacia de los fármacos están hechos con pacientes que tienen un diagnóstico claro.

\section{Desarrollo y perfeccionamiento de instrumentos de evaluación}

En los últimos años se ha incrementado el esfuerzo de los investigadores en lo referente al desarrollo y perfeccionamiento de instrumentos de evaluación que permitan al médico de APS identificar los distintos trastornos mentales, especialmente ansiedad, depresión y somatización. Además, se ha hecho más frecuente la utilización de instrumentos de screening y diagnóstico entre los MAP.

Por ejemplo, el cuestionario PRIME-MD es un instrumento válido y fiable, con una adecuada especificidad y sensibilidad que puede ayudar al médico tanto de AP como de otras especialidades medicoquirúrgicas a identificar los trastornos psiquiátricos más frecuentes ${ }^{55}$. Este instrumento dispone además de una versión computerizada que va guiando las preguntas del médico, pasando de unas preguntas generales que, en caso de ser respondidas afirmativamente, enlazan con una serie de módulos relacionados con los trastornos más frecuentes: ansiedad, depresión, somatización $y$ adicciones, entre otros.

Aunque la duración de la entrevista con el PRIMEMD no rebasa los 10 minutos, se han hecho esfuerzos por diseñar nuevos instrumentos o reducir el número de ítems de los ya existentes con el fin de ayudar al medico a detectar los casos en el mínimo tiempo posible. Por ejemplo, Means-Christensen, Arnau, Tonidandel, Bramson y Meagher ${ }^{56}$ indican que con un número pequeño de ítems es posible, con eficiencia y efectividad, detectar los casos de ansiedad y depresión que afectan a un porcentaje importante de los pacientes que acuden a las consultas de AP. Concretamente con cinco ítems del ADD (Detector de Depresión y Ansiedad) se puede detectar la depresión y/o ansiedad en AP ${ }^{57}$.

Otro instrumento ampliamente utilizado en AP es el HADS, ya que resulta adecuado para la detección de casos de depresión mayor y trastornos de ansiedad ${ }^{58}$. También en nuestro contexto se han desarrollado escalas para la evaluación en el contexto de AP de la depresión en el adulto ${ }^{59}$ o más específicamente en ancianos ${ }^{60}$. 
En resumen, se puede decir que para mejorar la atención que se presta a los usuarios de AP es necesario seguir trabajando en el desarrollo de instrumentos de evaluación y diagnóstico de los problemas de ansiedad y/o depresión. Un buen ejemplo de la importancia de este fenómeno es la aparición en el año 2000, editado por Mark E. Marvish, del primer manual de evaluación psicológica en atención primaria (Handbook of Psychological Assessment in Primary Care Settings $)^{61}$.

A modo de guía, podemos decir que las escalas de evaluación y diagnóstico de depresión y ansiedad, de más fácil y frecuente utilización, en función de su fiabilidad, especificidad y eficiencia, y que no exigen un entrenamiento especial para su aplicación e interpretación, son las siguientes (para más detalle ver Marvish, 2000): Escala de Ansiedad y Depresión de Goldberg, Escala de Hamilton para la Depresión (HDRS), Escala de Hamilton para la Ansiedad (HARS), Escala de Depresión de MontgomeryAsberg (MADRS), Escala Geriátrica abreviada de la Depresión de Yesavage. Otros cuestionarios útiles en Atención Primaria son: Escala Autoaplicada para la medida de la Depresión de Zung y Conde, Inventario de Depresión de Beck (BDI), Escala Breve de Ansiedad de Tyrer (BSA), Cuestionario de Screening de Ansiedad (ASQ-15), Patient Health Questionnaire (PHQ-9).

\section{Desarrollar y adaptar técnicas psicoterapéuticas útiles en el contexto de la AP}

Por otro lado, también se empiezan a desarrollar y adaptar técnicas psicoterapéuticas útiles en el contexto de la AP. Esta es una nueva fuente de investigación que se ha centrado fundamentalmente en la creación de herramientas terapéuticas para ayudar a los médicos de AP en el tratamiento de la ansiedad y la depresión. En general, las técnicas que se han desarrollado o adaptado giran en torno a alguna variación de la terapia cognitivo-conductual, ya que, según una reciente revisión de 16 metaanálisis metodológicamente rigurosos, este tipo de terapias confiere una gran ventaja frente a los tratamientos farmacológicos en una amplia gama de trastornos psicológicos. Este tratamiento psicológico es más eficaz (se halló un gran tamaño del efecto para los casos de trastorno depresivo mayor, trastorno de ansiedad generalizada, trastorno de pánico con y sin agorafobia, fobia social, trastorno por estrés postraumático, y trastornos de ansiedad en general) y produce menos abandonos que el tratamiento farmacológico $0^{62}$. Son las técnicas psicológicas cognitivo-conductuales basadas en la evidencia científica, que tras un entrenamiento progresivo (en relajación, reestructuración cognitiva, exposición, entrenamiento en habilidades sociales, etc.), consiguen eliminar los trastornos y síntomas derivados del estrés.

Por ejemplo, la Depression Management Tool Kit es una herramienta, revisada en 2004, que fue desarrollada como una iniciativa para ayudar a los médicos de AP en el tratamiento de las personas que sufren depresión. Los procesos de cuidados recomendados han sido desarrollados por la Agence for Healthcare Research and Quality y otras fuentes basadas en la evidencia (ver http:// www.depression-primarycare.org/).

Otra iniciativa interesante, para el tratamiento de la depresión y la prevención del suicido en ancianos, la representa el grupo PROSPECT (Prevention of Suicide in Primary Care Elderly: Collaborative Trial). En un reciente estudio se ha demostrado que este procedimiento basado en algoritmos reduce la mortalidad en pacientes ancianos con depresión mayor, después de cinco años desde el diagnóstico ${ }^{63,64}$.

En el apartado del tratamiento, se ha evaluado recientemente la relación coste-eficacia de un programa computarizado de intervención cognitivoconductual para el tratamiento de la depresión y la ansiedad en los centros de AP, denominado (Beating the Blues ${ }^{T M}$ ) con unos buenos resultados en comparación con el tratamiento habitual proporcionado por los equipos de $\mathrm{AP}^{65-67}$.

Beating the Blues ${ }^{\mathrm{TM}}$ es un programa computerizado de terapia cognitivo comportamental (CCBT) para el tratamiento de la depresión y la ansiedad. Es un programa clínicamente probado, libre de fármacos, que integra las terapias psicológicas con un avanzado software multimedia, en un tratamiento auto-administrado de 8 sesiones, diseñado para ser usado por personas sin conocimientos informáticos previos. Durante las sesiones los usuarios identifican problemas específicos y objetivos realistas. En los módulos cognitivos, los pacientes se centran en identificar y cambiar pensamientos automáticos, pensamientos erróneos, distracciones, creencias y estilos de atribución. Al mismo tiempo se incorporan componentes comportamentales basados en la solución realista de problemas, selección y trabajo con actividades placenteras, exposición graduada a determinadas situaciones o manejo de problemas de sueño, entre otros. El último modulo ayuda al paciente a establecer una planificación de la acción y prevenir las recaídas. Además utiliza módulos interactivos, animaciones y voces que motivan y refuerzan al paciente. La parte más importante del trabajo se basa en una serie de cortos que representan estudios de casos de ansiedad y depresión ficticios, que ayudan a demostrar el tratamiento cognitivo comportamental ${ }^{65,66}$. 
En la actualidad se han probado alternativas como la psicoterapia de grupo para los problemas de ansiedad y/o depresión derivados de la fibromialgia ${ }^{68}$ o la psicoterapia breve (4 sesiones) basada en la Revisión de Vida para el tratamiento de la depresión en ancianos ${ }^{69,70}$.

\section{Avances en el campo de la salud mental comunitaria}

Numerosos países han iniciado nuevas políticas y han creado y reformulado sus planes de salud para incluir el tratamiento de la salud mental, consiguiendo importantes avances en esta línea, como la incorporación del tratamiento de la enfermedad mental en los niveles de AP, la desinstitucionalización de los hospitales psiquiátricos, el aumento del número de profesionales dedicados a la salud mental y la mejora en la formación del personal sanitario en este tema, entre otros (ejemplos de estos recientes cambios los encontramos en Australia, India o Reino Unido).

Sin embargo, en lo que respecta a nuestro país, el panorama dista mucho de alcanzar los niveles europeos, particularmente cuando analizamos el número de recursos humanos (psiquiatras, psicólogos, enfermeros y trabajadores sociales) destinados a los servicios de salud mental. Así lo muestra el informe elaborado por la OMS en el Proyecto Atlas ${ }^{71-73}$.

Según este informe, los españoles tienen a su disposición 6,5 psiquiatras y 4,3 psicólogos por cada 100.000 habitantes, posicionándose muy por debajo del contexto europeo, que cuenta con 11 psiquiatras y 18 psicólogos por cada 100.000 habitantes, y alejándose hasta un $76 \%$ de la media europea para el caso de los profesionales de la Psicología. El déficit de enfermeros y de trabajadores sociales dedicados al área de la salud mental en nuestro país es también bastante alarmante, desviándose un $73,8 \%$ y un $95,7 \%$ por debajo de la media europea. Por tanto, una medida necesaria es el incremento paulatino del número de profesionales dedicados a la salud mental en el contexto del sistema público de salud, tanto en AP como en los servicios de salud mental ${ }^{73}$.

\section{CONCLUSIONES}

Después de todo lo expuesto anteriormente, existen una serie de conclusiones que podemos extraer en relación con la atención a los problemas de ansiedad y depresión en el contexto de AP.

En primer lugar, hay que señalar la importancia de la formación específica en estos temas de los MAP. Como ya hemos puesto de manifiesto, los diversos trabajos que han explorado esta cuestión han mostrado que, en general, un incremento en la formación específica conlleva una mejora sustancial sobre todo en lo que se refiere al correcto diagnóstico de los problemas de ansiedad y depresión. Y si el diagnóstico es acertado, el tratamiento, sobre todo farmacológico pero también en cuanto a orientación y consejo, ha mostrado ser tan efectivo como el proporcionado en los servicios especializados. Por tanto, se hace cada vez más necesario mejorar la capacidad de los médicos no psiquiatras para detectar las enfermedades mentales, ya que una vez detectadas el tratamiento suele ser efectivo. Para conseguirlo, una de las estrategias más importantes es el desarrollo de programas educativos dirigidos a los MAP.

En segundo lugar, la necesidad de hacer un esfuerzo colectivo a la hora de desarrollar instrumentos de evaluación que permitan al MAP hacer una aproximación diagnóstica con poco tiempo y un número reducido de ítems. En este campo se han hecho, con éxito notable, bastantes esfuerzos en los últimos años, por ejemplo para la evaluación de la depresión ${ }^{60} \mathrm{o}$ del insomnio ${ }^{74}$ en ancianos.

En tercer lugar, sería conveniente la investigación y el desarrollo de técnicas de psicoterapia específicas o adaptadas a su uso en el contexto de atención primaria. Este es un campo especialmente interesante pues desde el uso de las TIC hasta la psicoterapia breve ${ }^{69,70}$ o la terapia de grupo $^{68,75,76}$ pueden ser buenas alternativas. Por supuesto, sin olvidar la importancia del cálculo coste/beneficio a la hora de implementar las nuevas terapias. Esto exige un esfuerzo importante de investigación, pero creemos que puede ser, junto al desarrollo de instrumentos de evaluación, la línea de investigación preferente en el campo del manejo de los problemas de ansiedad y depresión en atención primaria. $Y$ este principio es especialmente importante en el área de la atención a los problemas de ansiedad y depresión en psicogeriatría.

En cualquier caso, el modelo de atención especializada de los problemas de salud mental no se puede exportar a los centros de salud ya que, teniendo en cuenta la demanda, se colapsaría en poco tiempo. Por tanto, el modelo de atención a la salud mental en AP tiene que ser específico, adaptados a las características sociosanitarias de los centros de salud, de los profesionales que allí trabajan y, por supuesto, de los usuarios.

\section{BIBLIOGRAFÍA}

1. Shear MK, Schulberg HC. Anxiety disorders in primary care. Bulletin of the Menninger Clinic. 1995; 59:73-85.

2. Jenkins R, Goldberg D, Kiima D, Mayeya J, Mayeya P, Mbatia J, Mussa M, Njenga F, Okonji M, Paton, J. Classification in primary care: Experience with current diagnostic systems. Journal of Psychopathology. 2002; 35:127-31.

3. Tizón JL. Medicina general, Psiquiatría y medicina psicosomática: Enfermos orgánicos y funcionales en la consulta de medicina general del seguro de enfermedad. 
Informaciones Psiquiátricas. 1973; 53:6-40.

4. Tizón JL, Ciurana R, Buitrago F, Camón R, Chocrón L, Fernández $\mathrm{C}$, et al. Prevención de los trastornos de la salud mental desde la Atención Primaria de salud. Revista de Atención Primaria. 1997; 20 (Supl 2):122-6.

5. Galeote P, Tizón JL, Spagnolo E, Pellegero N, Plans R. La cronificación del sufrimiento psicológico en la asistencia especializada ambulatoria de la Seguridad Social: Una perspectiva desde la Neuropsiquiatría de Zona. Psiquis. 1986; 71: 37-58.

6. Agüera LF, Reneses B, Muñoz PE. Enfermedad mental en Atención Primaria. Madrid: Flas; 1996.

7. Garrido S, Verdaguer S, Fuentes A,, Barbé E. Trastornos mentales en Atención Primaria de Salud. Revista de Atención Primaria. 1997; 19:499-500.

8. Baca BE, Sáiz RJ, Agüera Ortiz LF, Caballero ML, Fernández-Liria A, Ramos JA y cols. Prevalencia de los trastornos psiquiátricos en Atención Primaria usando el cuestionario PRIME-MD. Atención Primaria. 1999; 23:2759.

9. Iglesias GC, Díaz de la Peña A. Actitudes de los médicos de Atención Primaria sobre la salud mental en dos áreas sanitarias de Asturias. Anales de Psiquiatría. 1999; 15:18691.

10. Lopez-Torres J, Escobar F, Fernandez C, Gonzalez C, Urbistondo L, Requena M. Trastornos mentales y utilización de las consultas de medicina general. Atención Primaria. 1992; 10:35-40.

11. Goldberg D, Jemkins L, Millar T, Faragher B. The ability of general practitioners to identify emotional distress among their patients. Journal of Psychological Medicine. 1993; 23:185-93

12. Cano-Vindel A. Bases teóricas y apoyo empírico de la intervención psicológica sobre los desórdenes emocionales en Atención Primaria. Una actualización. Ansiedad y Estrés. 2011; 17:157-84

13. Cano-Vindel A. Los desórdenes emocionales en Atención Primaria. Ansiedad y Estrés. 2011; 17:73-95.

14. Cano Vindel A, Mae Wood C, Dongil E, Latorre JM. El trastorno de pánico en atención primaria. Papeles del Psicólogo. 2011; 32(3):265-73.

15. Shepherd M, Cooper B, Brown AC, Kalton GW. Psychiatric illness in general practice. London: Oxford University Press; 1966.

16. Baca E, Sáiz J, Porras A. Detección de trastornos mentales por médicos no psiquiatras: utilidad del cuestionario PRIME-MD. Medicina Clínica (Barc). 2001; 116:504-9.

17. Wells KB, Golding JM, Burnam MA. Psychiatric disorder in a sample of the general population with and without chronic medical conditions. American Journal of Psychiatry. 1988; 145:976-81.

18. Ceroni GB, Rucci P, Berardi D, Ceroni FB,, Katon W. Case review vs. usual care in primary care patients with depression: a pilot study. General Hospital Psychiatry. 2002; 24:71-80.

19. Szadoczky E, Rozsa S, Zambori J, Furedi J. Anxiety and mood disorders in Primary Care practice. Internacional Journal of Psychiatry in Clinical Practice. 2004; 8:77-84.

20. Kroenke K, Spitzer R, Williams J, Monahan P, Lowe B. Anxiety disorders in Primary Care: prevalence, impairment comorbidity, and detection. Annals of Internal Medicine. 2007; 146:317-25.

21. Latorre JM, López-Torres $\mathrm{J}$, Montañés $\mathrm{J}$, Parra $\mathrm{M}$. Percepción de la demanda y necesidad de formación de los médicos de atención primaria en salud mental. Atención Primaria. 2005; 36:85-92.

22. Robbins JM, Kirmayer LJ, Cathebras P, Yaffe MJ, Dworkind M. Physician characteristics and the recognition of depression and anxiety in primary care. Medical Care. 1994; 32:795-812.

23. Weisberg RB, Dyck I, Culpepper L, Keller MB. Psychiatric treatment in primary care patients with anxiety disorders: a comparison of care received from primary care providers and psychiatrists. American Journal of Psychiatry. 2007; 164:276-82

24. Gabarron HE, Vidal Royo JM, Haro Abad JM, Boix SI, Jover BA,, Arenas PM. Prevalencia y detección de trastornos depresivos en Atención Primaria. Atención Primaria. 2002; 29:329-36.

25. Johnstone A, Goldberg D. Psychiatric screening in general practice. A controlled trial. Lancet. 1976; 20:605-8.

26. Mitchell AL Vaze A, Rao S. Clinical diagnosis of depression in primary care: a meta-analysis. Lancet. 2009; 374:609-19.

27. Segui Díaz M, Linares Pou L, Blanco Lopez W, Ramos Aleixades J, Torrent Quetglas M. Tiempos durante la visita médica en atención primaria. Atención Primaria. 2004; 33:496-502

28. Deveugele,M, Derese A, van den Brink-Muinen A, Bensing J, De Maeseneer J. Consultation length in general practice: cross sectional study in six European countries. British Medical Journal. 2002; 325(7362):472.

29. Vazquez-Barquero JL, Garcia J, Simon JA, Iglesias C, Montejo J, Herran A et al. Mental health in primary care. An epidemiological study of morbidity and use of health resources. British Journal of Psychiatry. 1997; 170:529-35.

30. Vázquez-Barquero JL, Wilkinson G, Williams P, DiezManrique JF, Peña C. Mental health and medical consultation in primary care settings. Psychological Medicine. 1990; 20:681-94

31. Ronalds C, Kapur, N, Stone K, Webb S, Tomenson B, Creed F. Determinants of consultation rate in patients with anxiety and depressive disorders in primary care. Family Practice. 2002; 19:23-8.

32. Dowrick C Buchan I. Twelve moth outcome of depression in general practice: does detection or disclosure make a difference? British Medical Journal. 1995; 31:1274-6.

33. Sebastián GR, Molá G M, Barreto RP, Corral MME, Muñoz LJ, Boncompte VMP. Percepción de malestar psíquico por el médico en un área básica de salud. Revista de Atención Primaria. 1998; 22(8):491-6.

34. Wilmink FW, Ormel J, Giel R, Krol B, Lindeboom EG, van der Meer K, Soeteman JH. General practitioners' characteristics and the assessment of psychiatric illness. Journal of Psychiatric Research. 1989; 23:135-49.

35. Shedler J, Beck A, Bensen S. Practical Mental Health Assessment in Primary Care. Validity and Utility of the Quick PsychoDiagnostics Panel. Journal of Family Practice. 2000; 49:614-21.

36. Bridges K, Goldberg D (1992). Somatic presentation of depressive illness in primary care. Journal of Royal College of General Practice: Occasional Papers, 9-11.

37. García-Campayo J, Campos R, Marcos G, Pérez-Echeverría MJ, Lobo A. Somatization in primary care in Spain II: Differences between somatisers and psychologisers. British Journal of Psychiatry. 1996; 168:348-53.

38. Lobo A, Garcia-Campayo J, Campos R, Marcos G, PerezEcheverria MJ. Somatization in primary care in Spain I: Estimates of prevalence and clinical characteristics. Working Group for the Study of the Psychiatric and Psychosomatic Morbidity in Zaragoza. British Journal of Psychiatry. 1996; 168:344-8.

39. Mergl R, Seidscheck I, Allgaier AK, Moller H J, Hegerl U, Henkel V. Depressive, anxiety, and somatoform disorders in primary care: prevalence and recognition. Depression \& Anxiety. 2007; 24:185-95.

40. Bridges KW, Goldberg DP. Somatic presentation of DSM-III psychiatric disorders in primary care. Journal of Psychosomatic Research. 1985; 29:563-9.

41. Bridges K, Goldberg D, Evans B, Sharpe T. Determinants of somatization in primary care. Psychological Medicine. 1991; 21:473-83

42. Goldberg D. The management of anxious depression in primary care. Journal of Clinical Psychiatry. 1999; 60 Suppl 7:39-42. 
43. Means-Christensen AJ, Sherbourne CD, Roy-Byrne PP, Schulman MC, Wu J, Dugdale DC et al. In search of mixed anxiety-depressive disorder: a primary care study. Depression \& Anxiety. 2006; 23:183-9.

44. Sartorius N, Ustün TB, Lecrubier $Y$, Wittchen $H U$. Depression comorbid with anxiety: results from the WHO study on psychological disorders in primary health care. British Journal of Psychiatry Supplement. 1996; 30:38-43.

45. Coyne JC, Klinkman MS, Gallo JJ, Schwenk TL. Short-term outcomes of detected and undetected depressed primary care patients and depressed psychiatric patients. Journal of General Hospital Psychiatric. 1997; 19:333-43.

46. Aragonés E, Piñol JL, Labad A. Comorbilidad de la depresión mayor con otros trastornos mentales comunes en pacientes de atención primaria. Atención Primaria. 2009 41:545-51.

47. Fernandez A, Haro JM, Codony M, Vilagut G, MartinezAlonso $\mathrm{M}$, Autonell $\mathrm{J}$ et al. Treatment adequacy of anxiety and depressive disorders: primary versus specialized care in Spain. Journal of Affective Disorders. 2006; 96:9-20.

48. DiMatteo M, Haskard K, Williams S. Health beliefs, disease severity, and patient adherence: a meta-analysis. Medical Care. 2007; 45:521-8.

49. Ley P. Communicating with patients. Londres: Chapman, Hall; 1988.

50. Latorre JM, López-Torres J, Sánchez-Nuñéz,T, Serrano JP Montañés J, Escobar F. Primary care doctors' perception of treatment demand and need for training in drug addiction issues. Primary Care \& Community Psychiatry. 2007; 12:33-41.

51. Howe D. Detecting psychological distress: can general practitioners improve their own performance? British Journal of General Practice. 1996; 46:407-10.

52. Gómez-Restrepo C, Bohórquez-Peñaranda A, OkudaBenavides M, Gil-Laverde JF Sánchez-Díaz, N. Primary care physician satisfaction with patients diagnosed with depression: International Depression Project results from Colombia. Revista Brasileira de Psiquiatría. 2006; 28:2839.

53. Vicente B, Kohn R, Levav I, Espejo F, Saldivia S, Sartorius $\mathrm{N}$. Training primay care physicians in Chile in the diagnosis and treatment of depression. Journal of Affective Disorders. 2007; 98:121-7.

54. Secades R, Rodriguez-García E, Valderrey J, FernándezHermida J, Vallejo G, Jiménez-García J. El consumo de psicofármacos en pacientes que acuden a Atención Primaria en el Principado de Asturias (España). Psicothema. 2003; 15:650-5.

55. Baca E, Saiz, J, Agüera L, Caballero L, Fernández-Liria A, Ramos $\mathrm{J}$ et al. Validación de la versión española del PRIME-MD: un procedimiento para el diagnóstico de trastornos mentales en atención primaria. Actas Españolas de Psiquiatría. 1999; 27:375-83.

56. Means-Christensen AJ, Arnau RC, Tonidandel AM, Bramson $\mathrm{R}$, Meagher MW. An efficient method of identifying major depression and panic disorder in primary care. Journal of Behavioral Medicine. 2005; 28:565-72.

57. Means-Christensen AJ, Sherbourne,CD, Roy-Byrne PP, Craske MG, Stein MB. Using five questions to screen for five common mental disorders in primary care: diagnostic accuracy of the Anxiety and Depression Detector. General Hospital Psychiatry. 2006; 28:108-18.

58. Bunevicius A, Peceliuniene J, Mickuviene N, Valius L, Bunevicius R. Screening for depression and anxiety disorders in primary care patients. Depression \& Anxiety. 2007; 24(7):455-60.

59. Cordero-Villafafila A, Ramos-Brieva JA, Gutierrez-Labrador $\mathrm{R}$, Zamarro-Arranz ML. Un procedimiento breve y rápido para medir los síntomas depresivos en atención primaria. Actas Española de Psiquiatría. 2011; 39:45-8.

60. López-Torres J, Galdón Blesa P, Fernández-Olano C Escobar-Rabadán F, Montoya Fernández J, Boix Gras C, et al. Diseño y validación de un cuestionario para la detección de depresión mayor en pacientes ancianos. Gaceta Sanitaria. 2005; 19:103-12.

61. Marvish ME (Ed). Handbook of Psychological Assessment in Primary Care Settings. Mahwah, NJ, LEA; 2000.

62. Butler AC, Chapman JE, Forman EM, Beck AT. The empirical status of cognitive-behavioral therapy: a review of meta-analyses. Clin Psychol Rev. 2006; 26:17-31.

63. Bruce ML, Ten Have TR, Reynolds CF, III, Katz II, Schulberg HC, Mulsant BH et al. Reducing suicidal ideation and depressive symptoms in depressed older primary care patients: a randomized controlled trial. JAMA. 2004; 291:1081-91.

64. Gallo JJ, Bogner HR, Morales KH, Post EP, Lin JY, Bruce $M L$. The effect of a primary care practice-based depression intervention on mortality in older adults: a randomized trial. Annals of Internal Medicine. 2007; 146:689-98.

65. Proudfoot J, Goldberg D, Mann A, Everitt B, Marks I, Gray JA. Computerized, interactive, multimedia cognitivebehavioural program for anxiety and depression in general practice. Psychological Medicine. 2003; 33:217-23.

66. Proudfoot J, Ryden C, Everitt B, Shapiro D A, Goldberg D, Mann A et al. Clinical efficacy of computerized cognitivebehavioral therapy for anxiety and depression in primary care: randomized controlled trial. British Journal of Psychiatry. 2004; 185:46-54.

67. McCrone P, Knapp M, Proudfoot J, Ryden C, Cavanagh K, Shapiro D A et al. Cost-effectiveness of computerized cognitive-behavioural therapy for anxiety and depression in primary care: randomized controlled trial. British Journal of Psychiatry. 2004; 185:55-62.

68. Parra M. Eficacia de la terapia cognitive basada en la conciencia plena (mindfulness) en pacientes con fibromialgia. Tesis Doctoral no publicada (2011). Universidad de Castilla-La Mancha, Albacete.

69. Serrano JP, Latorre JM, Gatz M, Montañés J. Life Rewiew therapy using autobiographical retrieval practice for older adults with depressive symptomatology. Psychology and Aging. 2004; 19:272-7

70. Serrano JP, Latorre JM, Ros L, Navarro B, Aguilar MJ, Nieto M, Ricarte JJ. Life Review therapy using autobiographical retrieval practice for older adults with clinical depression: results of a randomized clinical trial. Psicothema (en prensa).

71. Saraceno B, Saxena S. Mental health resources in the world: results from Project Atlas of the WHO. World Psychiatry. 2002; 1:40-4.

72. Saxena,S, Maulik,PK, O'Connell K, Saraceno B. Mental health care in primary and community settings: results from WHO's Project Atlas. International Journal of Social Psychiatry. 2002; 48:83-5.

73. Wahlbeck K. European comparisons between mental health services. Epidemiology and Psychiatric Sciences. $2011 ; 20: 15-8$

74. Navarro B. Estudio del insomnio en las personas mayores. Diseño y validación de la escala IPM (Insomnio en Personas Mayores). Tesis Doctoral no publicada (2011). Universidad de Castilla-La Mancha, Albacete.

75. Latorre JM, Hernández-Viadel, Ricarte JJ. Efficacy of group memory training method for older adults base don visualization and association techniques: a randomized, controlled trial with a placebo group. Applied Cognitive Psychology. 2010; 24:956-68.

76. Ricarte JJ, Hernández-Viadel JV, Latorre JM, Ros, L (2011). Effects of event specific-memory training on autobiographical memory retrieval and depressive symptoms in schizophrenic patients. Journal of Behavior Therapy and Experimental Psychiatry. 2011; Available online 23 June 2011, DOI: 10.1016/j.jbtep.2011.06.001. 